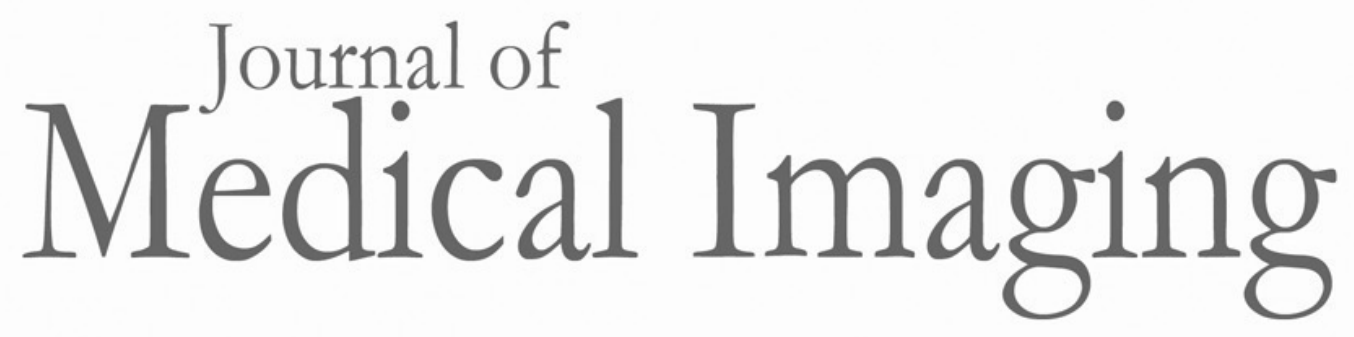

Medicallmaging.SPIEDigitalLibrary.org

\title{
Cardiac fiber tracking on super high-resolution CT images: a comparative study
}

Hirohisa Oda

Holger R. Roth

Takaaki Sugino

Naoki Sunaguchi

Noriko Usami

Masahiro Oda

Daisuke Shimao

Shu Ichihara

Tetsuya Yuasa

Masami Ando

Toshiaki Akita

Yuji Narita

Kensaku Mori 


\title{
Cardiac fiber tracking on super high-resolution CT images: a comparative study
}

\author{
Hirohisa Oda, ${ }^{\mathrm{a}, *}$ Holger R. Roth, ${ }^{\mathrm{a}}$ Takaaki Sugino, ${ }^{\mathrm{a}}$ Naoki Sunaguchi, ${ }^{\mathrm{b}}$ \\ Noriko Usami, ${ }^{c}$ Masahiro Oda, ${ }^{a}$ Daisuke Shimao, ${ }^{d}$ Shu Ichihara, \\ Tetsuya Yuasa, ${ }^{\mathrm{f}}$ Masami Ando, ${ }^{\mathrm{g}}$ Toshiaki Akita, ${ }^{\mathrm{c}}$ Yuji Narita, ${ }^{\mathrm{c}}$ and \\ Kensaku Mori ${ }^{\text {a,h,i }}$ \\ ${ }^{a}$ Nagoya University, Graduate School of Informatics, Nagoya, Japan \\ ${ }^{b}$ Nagoya University Graduate School of Medicine, Department of Radiological and \\ Medical Laboratory Sciences, Nagoya, Japan \\ ${ }^{c}$ Nagoya University School of Medicine, Department of Tissue Engineering, Nagoya, Japan \\ ${ }^{\mathrm{d}}$ Hokkaido University of Science, Department of Radiological Technology, Sapporo, Japan

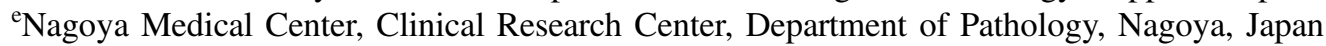 \\ fYamagata University, Graduate School of Engineering and Science, Yamagata, Japan \\ ${ }^{g}$ Tokyo University of Science, Research Institute of Science and Technology, Tokyo, Japan \\ ${ }^{\mathrm{h}}$ Nagoya University, Information Technology Center, Nagoya, Japan \\ iNational Institute of Informatics, Research Center for Medical Bigdata, Tokyo, Japan
}

\begin{abstract}
Purpose: High-resolution cardiac imaging and fiber analysis methods are required to understand cardiac anatomy. Although refraction-contrast x-ray CT (RCT) has high soft tissue contrast, it cannot be commonly used because it requires a synchrotron system. Microfocus X-ray CT $(\mu \mathrm{CT})$ is another commercially available imaging modality.

Approach: We evaluate the usefulness of $\mu \mathrm{CT}$ for analyzing fibers by quantitatively and objectively comparing the results with RCT. To do so, we scanned a rabbit heart by both modalities with our original protocol of prepared materials and compared their image-based analysis results, including fiber orientation estimation and fiber tracking.

Results: Fiber orientations estimated by two modalities were closely resembled under the correlation coefficient of 0.63 . Tracked fibers from both modalities matched well the anatomical knowledge that fiber orientations are different inside and outside of the left ventricle. However, the $\mu \mathrm{CT}$ volume caused incorrect tracking around the boundaries caused by stitching scanning.

Conclusions: Our experimental results demonstrated that $\mu \mathrm{CT}$ scanning can be used for cardiac fiber analysis, although further investigation is required in the differences of fiber analysis results on RCT and $\mu \mathrm{CT}$.

(C) The Authors. Published by SPIE under a Creative Commons Attribution 4.0 Unported License. Distribution or reproduction of this work in whole or in part requires full attribution of the original publication, including its DOI. [DOI: 10.1117/1.JMI.7.2.026001]
\end{abstract}

Keywords: high-resolution cardiac imaging; heart staining protocols; microtomography; fine anatomical structure analysis.

Paper 19044RR received Feb. 13, 2019; accepted for publication Feb. 11, 2020; published online Mar. 11, 2020.

\section{Introduction}

Deep understanding of the cardiac fiber structure in the left ventricle (LV) is required to understand cardiac anatomy and such diseases as heart failure. 6.5 million people experienced heart failure between 2011 and 2014 in the United States. ${ }^{1}$ Although the fiber structure may also be changed by heart failure, the details have not been clearly investigated yet.

*Address all correspondence to Hirohisa Oda, E-mail: hoda@mori.m.is.nagoya-u.ac.jp 
High-resolution cardiac imaging and analysis methods in three-dimensional (3-D) space are needed.

Diffusion tensor magnetic resonance imaging (DT-MRI) is well-known for analyzing cardiac fiber structure..$^{2-4}$ With DT-MRI, we estimate the fiber orientation at a point as the orientation with the strongest diffusion of water molecules. However, its resolution is inadequate. For instance, Helm et al. ${ }^{3}$ used a 1.5-T CV/I MRI scanner (General Electric) whose resolutions were 300,300 , and $800 \mu \mathrm{m}$ for each of three axes. Histopathological images have also been used ${ }^{5,6}$ for cardiac imaging with much higher resolution than DT-MRI. However, precise reconstruction of the heart's stacked section images is complicated due to the tissue damage caused by cutting the sections and the banana problem. ${ }^{7}$ 3-D analysis from a heart's histopathological stacks is very difficult.

We explored two alternate scanning modalities: refraction-contrast x-ray CT (RCT) and microfocus $\mathrm{x}$-ray $\mathrm{CT}(\mu \mathrm{CT})$. $\mathrm{RCT}^{8,9}$ is a 3-D imaging modality that is one type of phase-contrast CT scanning based on observing the refraction of x-rays. It has very high soft tissue contrast, even for cardiac fibers. However, RCT is not commercially available and cannot be utilized publicly because it requires a synchrotron system.

$\mu \mathrm{CT}$ is a commercially available 3-D imaging modality. In general, scanning is done by observing the absorption of x-rays that run through target objects. Resolution, contrast, and image size vary, as do their price ranges. Some scanners, which also observe phase shift, have very high resolution; SCYSCAN 1727 (Bruker) has the highest: 0.35- $\mu \mathrm{m}$ resolution. We utilize a relatively low-end type of scanner, inspeXio SMX-90CT Plus (Shimadzu, Japan), which only observes X-ray absorption; its highest resolution is around $5 \mu \mathrm{m}$. Nevertheless, although cardiac fibers can be observed on the $\mu \mathrm{CT}$ volumetric images (volumes) produced by this scanner, their contrast is not as clear as RCT volumes.

In this paper, we first describe our fiber analysis methods from the RCT or $\mu \mathrm{CT}$ volumes of the heart. Then, we analyze how $\mu \mathrm{CT}$ produces proper results by qualitatively and quantitatively comparing it with RCT. Fiber analysis consists of the estimation of orientation and tracking fibers and compares the results from a $\mu \mathrm{CT}$ volume with those of an RCT volume. We prepared a heart specimen with our original protocols and scanned it with RCT and $\mu \mathrm{CT}$ and registered their volumes. Using these registered RCT and $\mu \mathrm{CT}$ volumes, we compared the fiber orientation estimation results on a slice to check quantitatively whether the $\mu \mathrm{CT}$ volume produced similar fiber orientation estimation results as the RCT volume. We tracked the fibers to investigate whether fiber orientation can be estimated well on the $\mu \mathrm{CT}$ volume in the entire LV. Our experimental results demonstrated that $\mu \mathrm{CT}$ scanning can be used for cardiac fiber analysis, although further investigation is required of the differences of the fiber analysis results on RCT and $\mu \mathrm{CT}$. This paper is an extended version of our 2019 SPIE Proceedings paper. $^{10}$

\section{Fiber Analysis Method}

\subsection{Overview}

Our fiber analysis method consists of the following two schemes: (1) estimation of fiber orientation and (2) fiber tracking.

We did scheme (1) for each voxel in the input CT volume to estimate the fiber orientation around the voxels to quantitatively analyze the fiber orientation statistics.

We did scheme (2) on the entire CT volume to produce trajectories that follow the fibers. Scheme (1) must be performed during the tracking process. The results of scheme (2) are useful for qualitatively visualizing how fibers flow in the entire LV.

\subsection{Fiber Orientation Estimation}

Structure tensor (ST) analysis is commonly used for estimating the cardiac fiber orientation in $\mu \mathrm{CT}$ volumes. ${ }^{11,12}$ First, for each volume, we apply a Gaussian smoothing filter with standard deviation $\sigma_{\mathrm{P}}$ to smooth the intensity gradients and empirically set $\sigma_{\mathrm{P}}=20 \mu \mathrm{m}$. 
ST $\mathbf{T}(\mathbf{x})$ at voxel $\mathbf{x}$ is defined:

$$
\mathbf{T}(\mathbf{x})=\sum_{\mathbf{x}^{\prime} \in \mathcal{N}} w\left(\sigma_{\mathrm{T}},\left\|\mathbf{x}-\mathbf{x}^{\prime}\right\|\right) \mathbf{g}\left(\mathbf{x}^{\prime}\right) \mathbf{g}^{\mathrm{T}}\left(\mathbf{x}^{\prime}\right),
$$

where $\mathcal{N}$ is a set of the neighboring voxels around $\mathbf{x}, \mathbf{x}^{\prime}$ is one of the voxels in $\mathcal{N}, w\left(\sigma_{\mathrm{T}},\left\|\mathbf{x}-\mathbf{x}^{\prime}\right\|\right)$ is the Gaussian weight with standard deviation $\sigma_{\mathrm{T}}$ and distance $\left\|\mathbf{x}-\mathbf{x}^{\prime}\right\|$ from the center, and $\mathbf{g}\left(\mathbf{x}^{\prime}\right)$ is a local intensity gradient vector around $\mathbf{x}^{\prime}$. T $(\mathbf{x})$ can be written as a $3 \times 3$ matrix. The eigenvector of $\mathbf{T}(\mathbf{x})$, which corresponds to smallest eigenvalue $\mathbf{f}(\mathbf{x})$, is assumed to be a direction of the fiber orientation at $\mathbf{x}$, which has the smallest intensity changes around $\mathbf{x}$. We set $\sigma_{\mathrm{T}}$ to $400 \mu \mathrm{m}$.

\subsection{Fiber Tracking}

We randomly generated $N$ initial points in the mask of the LV region. From each initial point, fiber tracking was done by an iterative process. First (iteration $k=0$ ), we estimated fiber direction vector $\mathbf{f}\left(\mathbf{x}_{\mathbf{0}}\right)$ at each initial point $x_{\mathbf{0}} \in \mathbf{R}^{3}$ using the ST analysis described in Sec. 2.2. Since the fibers are running in both directions, $\mathbf{f}\left(\mathbf{x}_{0}\right)$ and $-\mathbf{f}\left(\mathbf{x}_{0}\right)$, fiber tracking was also performed for both directions. We calculated the endpoint coordinates of the trajectories at the $k^{\prime}$ th iteration (iteration $k>0$ ):

$$
\begin{aligned}
\mathbf{x}_{k} & =\mathbf{x}_{k-1}+s \mathbf{f}\left(\mathbf{x}_{k-1}\right), \\
\mathbf{x}_{-k} & =\mathbf{x}_{-(k-1)}-s \mathbf{f}\left[\mathbf{x}_{-(k-1)}\right],
\end{aligned}
$$

where $s$ represents the step size, $\mathbf{f}\left(\mathbf{x}_{k-1}\right)$ represents the orientation vector at $\mathbf{x}_{k-1}$, and $\mathbf{f}\left[\mathbf{x}_{-(k-1)}\right]$ represents the orientation vector at $\mathbf{x}_{-(k-1)}$. We terminated the tracking for each direction when $\mathbf{x}_{k}$ or $\mathbf{x}_{-k}$ was outside the LV mask, or index $k$ of the iterations reached $k_{\max }$. We set the parameters to $n_{\mathrm{i}}=1000, s=4$ voxels, and $k_{\max }=1000$. The trajectories, which were tracked from all the initial points, are output.

\section{Materials}

\subsection{RCT and $\mu \mathrm{CT}$ Scanning}

RCT and $\mu \mathrm{CT}$ volumes of a rabbit heart were obtained by the following sequence: (1) harvesting a heart, (2) ethanol fixation, (3) RCT scanning, (4) contrast enhancement, (5) rinse, and (6) $\mu \mathrm{CT}$ scanning. Fixation was performed once using ethanol. $\mathrm{I}_{2} \mathrm{KI}$ was used for the contrast enhancement for the $\mu \mathrm{CT}$ scanning. Ethanol was used again in preparation for the $\mu \mathrm{CT}$ scanning for rinsing excess $\mathrm{I}_{2} \mathrm{KI}$ to reduce the artifact.

The following are the specimen preparation and scanning procedures. We scanned one $\mu \mathrm{CT}$ and one RCT volume of a rabbit heart (Fig. 1) under the IRB approval of Nagoya University.

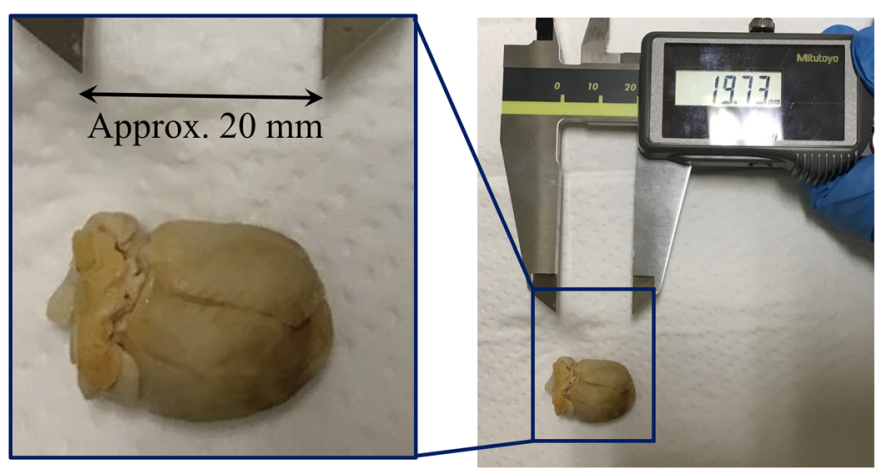

Fig. 1 Rabbit heart: longest axis is about $20 \mathrm{~mm}$. 


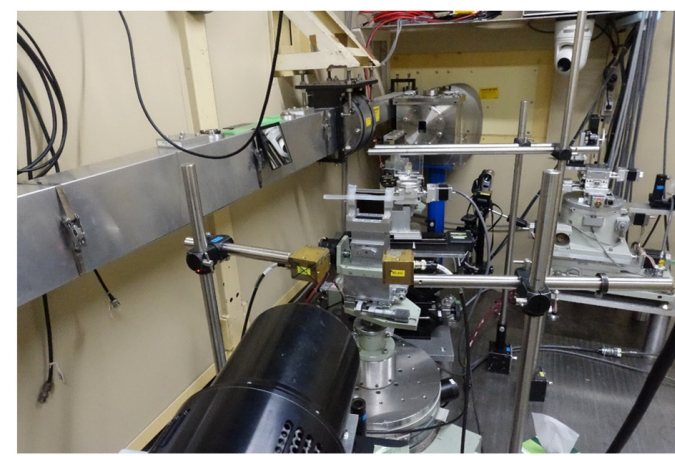

(a) RCT

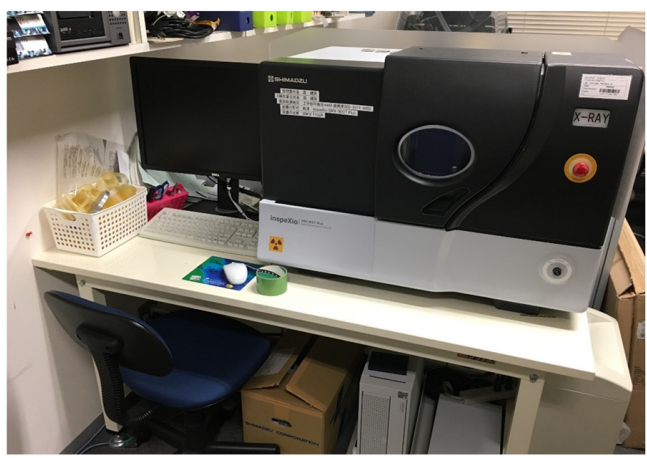

(b) $\mu \mathrm{CT}$

Fig. 2 Machines that scanned rabbit heart in Fig. 1: (a) RCT and (b) $\mu \mathrm{CT}$.

We harvested the heart of a Japanese white rabbit (10-week-old male) just after euthanasia with a $\mathrm{KCl}$ injection into the aortic arch and obtained a heart specimen. The following is the RCT scanning procedure: (1) ethanol fixation: the heart was fixated with an $80 \%$ ethanol water solution since ethanol fixation effectively improves the tissue contrast better than formalin fixation for the other phase-contrast imagings of hearts. ${ }^{13}$ (2) RCT scanning: RCT scanning was performed using the synchrotron system developed by Ando et al.'s group [Fig. 2(a)] at the High Energy Accelerator Research Organization (KEK) (Japan). ${ }^{14}$ The synchrotron system used for RCT scanning cost about 177 million USD ( 1 USD $=110$ JPY $).{ }^{15}$ The RCT scanning specifications are listed in Table 1. Axial and coronal slices of the RCT volume are shown in Fig. 3(a).

After RCT scanning, we scanned the same heart specimen in the following manner. We introduced an additional staining process for $\mu \mathrm{CT}$ scanning. (1) Contrast enhancement: We stained the rabbit heart with a 7.5\% $\mathrm{I}_{2} \mathrm{KI}$ solution for one day. (2) Rinse: the heart was briefly rinsed in an $80 \%$ ethanol solution. (3) $\mu \mathrm{CT}$ scanning. Table 2 shows the scanning specification. Our scanner's field of view (FOV) was limited: $1024 \times 1024 \times 548$ voxels at $17 \times 17 \times 17 \mu \mathrm{m}^{3} /$ voxel resolution. It has a stitch-scanning mode to cover larger FOVs. We used this feature to cover the entire heart (three consecutive scans), although not every volume was aligned well in the stitching mode. Furthermore, ring artifacts on the $\mu \mathrm{CT}$ volume were quite obvious. We used TomoPy ${ }^{16}$ to reduce the ring artifacts, which are commonly observed on $\mu \mathrm{CT}$ volumes. Examples of the axial and coronal slices of the $\mu \mathrm{CT}$ volume are shown in Fig. 3(b).

This work used a desktop-type $\mu \mathrm{CT}$ scanner, inspeXio SMX-90CT Plus (Shimadzu, Japan) [Fig. 2(b)], which is a low-end, desktop type. Its catalog price is $\sim 236,000$ USD $(1 \mathrm{USD}=110 \mathrm{JPY})$. Ethanol fixation ${ }^{13}$ is also suitable for $\mu \mathrm{CT}$ scanning in combination with contrast enhancement. Other $\mu \mathrm{CT}$ cardiac imaging works ${ }^{12,17}$ use high-end, much more expensive $\mu \mathrm{CT}$ scanners than ours. In those works, contrast enhancement continued for several days by staining the specimens in an iodine-potassium iodide $\left(\mathrm{I}_{2} \mathrm{KI}\right)$ solution. For instance, one trial by

Table 1 RCT scanning specifications.

\begin{tabular}{|c|c|}
\hline Item & Value \\
\hline Location for scanning & $\begin{array}{l}\text { Photon Factory, High Energy Accelerator Research Organization } \\
\text { (Tsukuba, Japan) }\end{array}$ \\
\hline Camera & VHR 16 M (Photonics Science) \\
\hline X-ray optical system & X-ray dark field imaging \\
\hline Resolution & $15 \times 15 \times 15 \mu \mathrm{m}^{3} /$ voxel \\
\hline Volume size & $1600 \times 1600 \times 1240$ voxels \\
\hline X-ray energy & $19.8 \mathrm{keV}$ \\
\hline
\end{tabular}



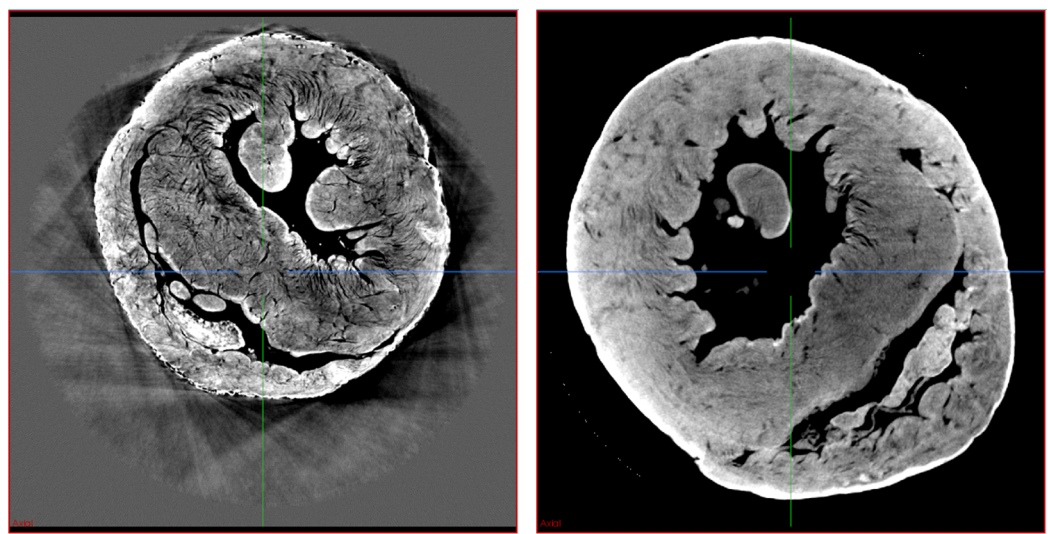

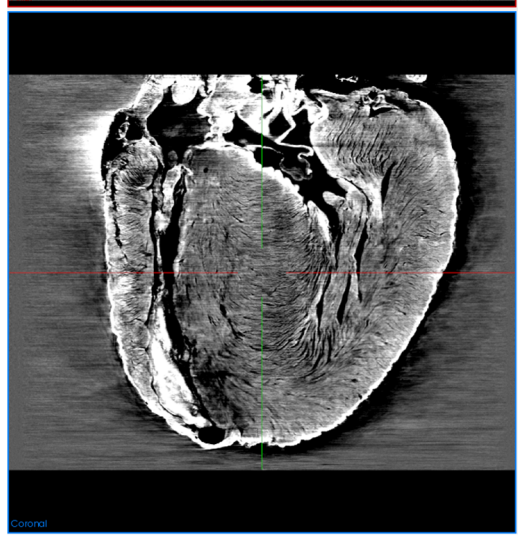

(a) RCT

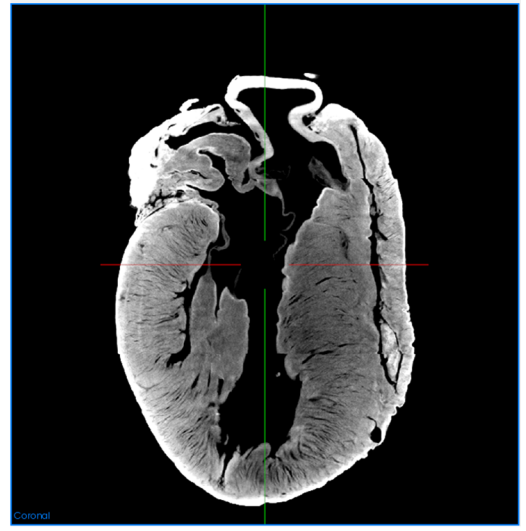

(b) $\mu \mathrm{CT}$

Fig. 3 Axial and coronal slices: (a) RCT and (b) $\mu \mathrm{CT}$ volumes. Fibers on RCT volume look clearer than those of $\mu \mathrm{CT}$ volume. Registration is required for comparison due to different heart positions.

Table 2 Specifications of $\mu \mathrm{CT}$ scanning.

\begin{tabular}{ll}
\hline \hline Item & \multicolumn{1}{c}{ Value } \\
\hline Location for scanning & Nagoya University (Nagoya, Japan) \\
Scanner & inspeXio SMX-90CT Plus (Shimadzu) \\
Resolution & $17 \times 17 \times 17 \mu \mathrm{m}^{3} / \mathrm{voxel}$ \\
Volume size & $1024 \times 1024 \times 1627$ voxels \\
\# of divided-scanning parts & 4 \\
Tube voltage & $90 \mathrm{kVp}$ \\
Tube current & $110 \mu \mathrm{A}$ \\
\# of x-ray projection & 1200 \\
\# of projections for each angle & 12 \\
\hline \hline
\end{tabular}

Stephenson et al. ${ }^{17}$ stained a rabbit heart in a $7.5 \% \mathrm{I}_{2} \mathrm{KI}$ solution for 3 days with the Metris X-Tec custom $320-\mathrm{kV}$ bay system with $155-\mathrm{kV}$ tube voltage and $150-\mu \mathrm{A}$ tube current. However, directly using the same protocols as these Refs. 12 and 17 for our scanner caused artifacts since our scanner has lower x-ray energy.

RCT has superior soft tissue contrast to $\mu \mathrm{CT}$. This means that RCT can depict different soft tissues in different intensities although $\mu \mathrm{CT}$ depicts such soft tissues in the same intensities. Phase-contrast x-ray imaging including RCT has been developed for better soft tissue contrast. 


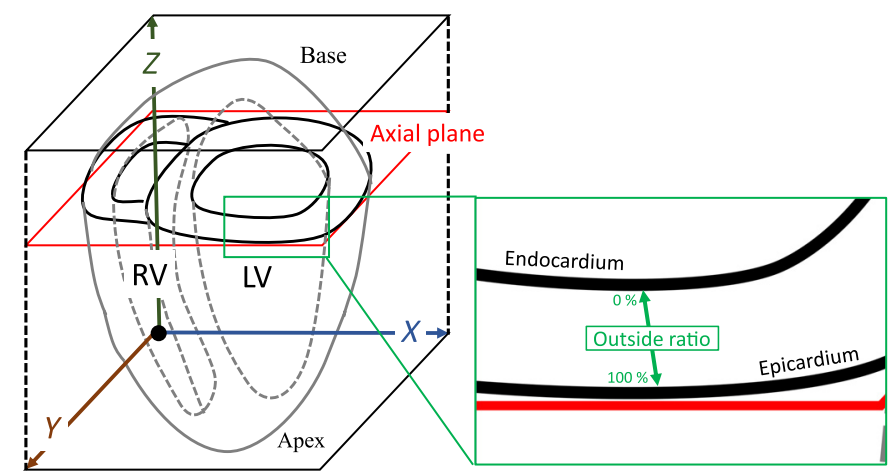

Fig. 4 Coordinate system and position of ventricles: Axial planes $(x-y$ plane) cut axis along base and apex into rounds. On axial planes, RV is shown on left of LV. Outside ratio is illustrated in magnified part. Outside ratio becomes $0 \%$ at endocardium side and $100 \%$ at epicardium side.

\subsection{Registration of $\mu \mathrm{CT}$ and RCT Volumes}

To compare the fiber analysis results, we registered the RCT volume as $\mu \mathrm{CT}$. The heart's $\mu \mathrm{CT}$ and RCT volumes were cropped and rotated manually using the MITK Workbench 2016.11. ${ }^{18}$ The LV is entirely covered with a slight margin around it and roughly aligned between the two volumes whose size and resolution were adjusted into $900 \times 980 \times 1080$ and $18 \times 18 \times$ $18 \mu \mathrm{m} /$ voxels respectively. The coordinate system of these volumes is shown in Fig. 4. Since the parts of the surrounding regions such as RV were also included, the processing target region was specified by masking. The mask of the LV region (LV mask) was segmented semiautomatically using the MITK Workbench $2016.11^{18}$ on the $\mu \mathrm{CT}$ volume. Then, we applied nonrigid registration to the RCT volume to align it with the $\mu \mathrm{CT}$ volume. We used deedsBCV, which is opensource software published by Heinrich et al. ${ }^{19}$

Figures 5(a) and 5(b) show the axial and coronal slices of the registration results. In Fig. 5(c), the axial slices of the two registered volumes are shown as one figure after being merged to

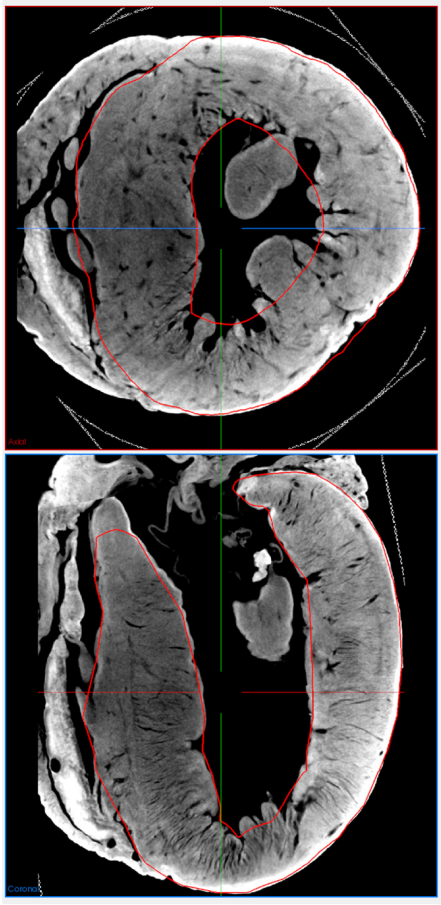

(a) RCT

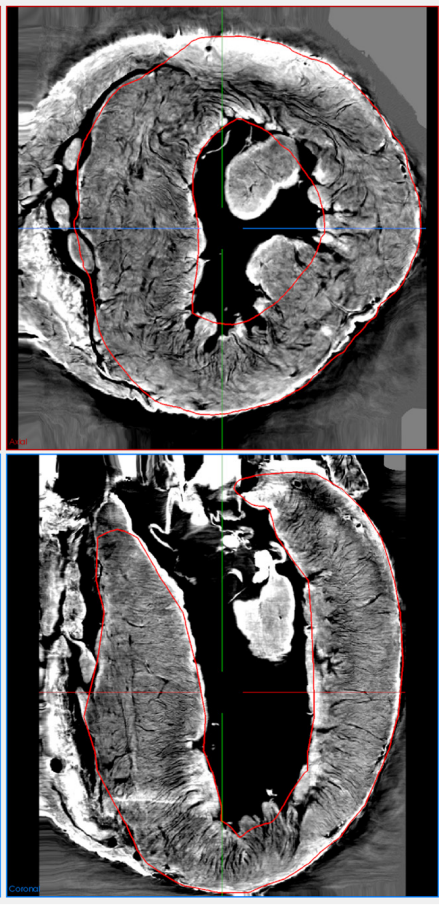

(b) $\mu \mathrm{CT}$

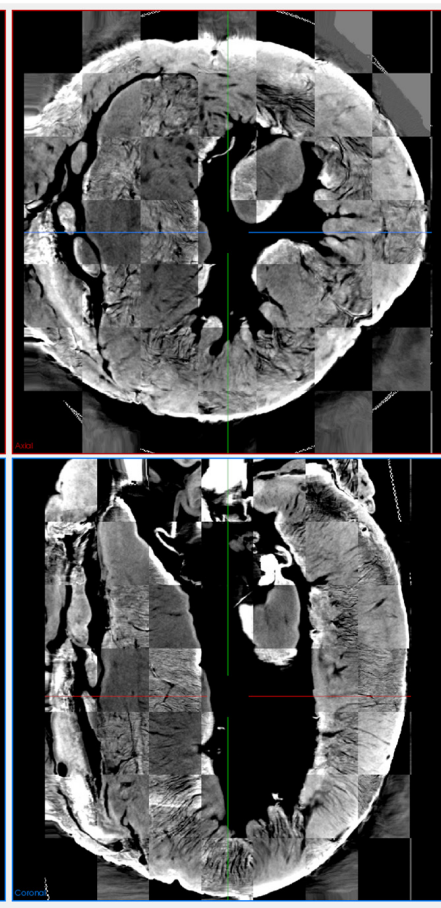

(c) Registered volume

Fig. 5 Axial and coronal slices of registered volumes: Registration results of (a) RCT and (b) $\mu$ CT with LV mask (red line); (c) checkerboard-like scheme visualization of these volumes. 
resemble a checkerboard. Clearly, the RCT volume was successfully registered to the $\mu \mathrm{CT}$ volume. As shown in Fig. 5(c), the boundaries of the LV and the image patterns shown in both volumes were successfully aligned.

\section{Experimental Setup}

\subsection{Overview}

We evaluated how our fiber analysis method produced precise results from the $\mu \mathrm{CT}$ volume by comparing them with the RCT volume results. We performed fiber tracking for each registered volume to compare the tracking results obtained from the RCT and $\mu \mathrm{CT}$ volumes. We analyzed the fiber orientation statistics on multiple axial slices. Detailed analysis was conducted on one of those axial slices around the central part of the LV and focused on fiber orientations. The 3-D visualization of the fibers was performed by fiber tracking (Sec. 2.3).

\subsection{Fiber Orientation Statistics}

\subsubsection{Definition of outside ratio}

Anatomical studies ${ }^{5,20}$ clarified that inside and outside of the LV tends to have different fiber orientations. Therefore, analyzing the fiber orientations may produce different results that correspond to their respective positions inside and outside the LV. We define the outside ratio measure based on whether each sample point is represented as nearer the outside of the LV wall than inside it. The outside ratio becomes $0 \%$ at the endocardium side and $100 \%$ at the epicardium side, as illustrated in the magnified part of Fig. 4.

From the center point of the LV region on an axial slice, we performed radial searches to eight angles on an axial slice. On each search, we obtained a set of sample points whose outside ratios were $10 \%, 20 \%, \cdots$, or $90 \%$. On each sample point, we individually estimated the fiber orientation from the RCT and $\mu \mathrm{CT}$ volumes, where the axial slices cut the heart orthogonally to its longest axis (Fig. 4).

\subsubsection{Angle difference of $\mu \mathrm{CT}$ from $R C T$}

We define the angle difference of $\mu \mathrm{CT}$ from RCT $\theta_{1}$ :

$$
\theta_{1}=\cos ^{-1}\left\{\mathbf{f}^{\mu}(\mathbf{x}) \cdot \mathbf{f}^{\mathrm{R}}(\mathbf{x})\right\} \quad\left(0 \leq \theta_{1} \leq \pi\right)
$$

where $\mathbf{f}^{\mu}(\mathbf{x})$ and $\mathbf{f}^{\mathrm{R}}(\mathbf{x})$ represent the unit vectors of the fiber orientations estimated from the $\mu \mathrm{CT}$ and RCT volumes [Fig. 6(a)], respectively. Assuming the orientation from RCT is the groundtruth, the angle difference of $\mu \mathrm{CT}$ from RCT represents the estimation error on $\mu \mathrm{CT}$.

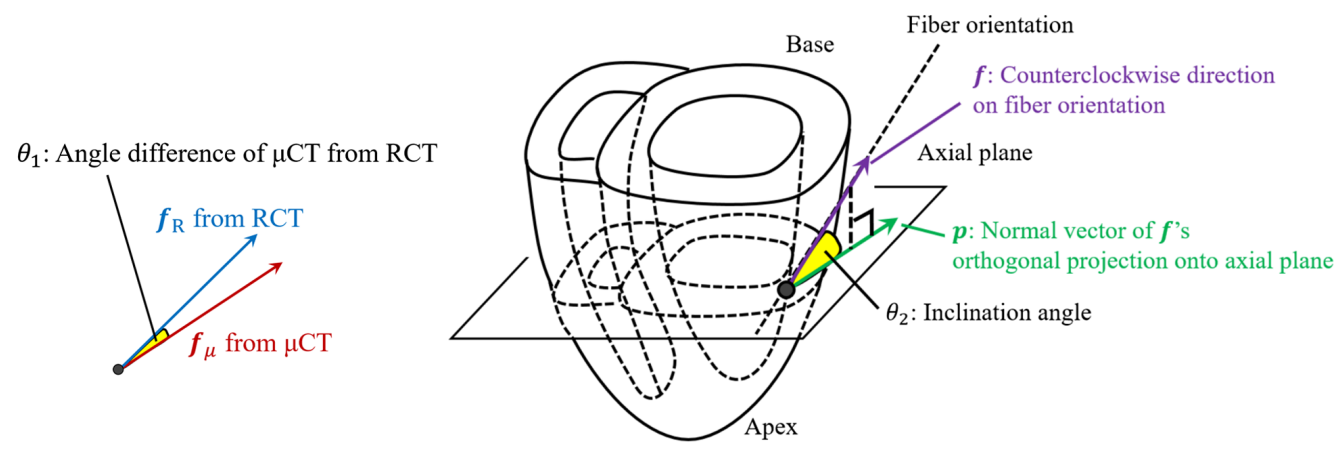

(a) Angle difference of $\mu \mathrm{CT}$ from RCT

(b) Inclination angle

Fig. 6 Definitions of angles: (a) angle difference of $\mu \mathrm{CT}$ from RCT $\theta_{1}$ and (b) inclination angle $\theta_{2}$. 
To evaluate how the fiber orientations estimated from $\mu \mathrm{CT}$ volumes are different from those of the RCT, we computed the average and standard deviations of the angle difference of $\mu \mathrm{CT}$ from RCT at 100-slice intervals and plotted them on a graph. We also visualized the angle differences of $\mu \mathrm{CT}$ from RCT on sample points on an axial slice around the central area.

\subsubsection{Inclination angle}

Inclination angle $\theta_{2}$ follows anatomical studies. Streeter et al..$^{5}$ defined fiber angle $\alpha$ and showed that it becomes positive inside and negative outside the LV. Our definition of inclination angle resembles their definition, which can be computed in 3-D volumes. As shown in Fig. 6(b), the inclination angle is defined as

$$
\theta_{2}=\cos ^{-1}\{\mathbf{f}(\mathbf{x}) \cdot \mathbf{p}\} \quad\left(-\pi<\theta_{2} \leq \pi\right),
$$

where $\mathbf{f}(\mathbf{x})$ represents the estimated fiber orientation. $\mathbf{p}=\frac{p[\mathbf{f}(\mathbf{x})]}{\|p[\mathbf{f}(\mathbf{x})]\|}$ represents a unit vector on the axial plane, written by orthographic projection $p[\mathbf{f}(\mathbf{x})]$ of $\mathbf{f}(\mathbf{x})$ onto the axial plane.

We visualized the angle difference of $\mu \mathrm{CT}$ from RCT of each sample point on an axial slice around the central area. We drew a scatter plot of the inclination angles computed from $\mu \mathrm{CT}$ and RCT and verified the statistical significance of the correspondence. We also observed the correlation between the outside ratio and the inclination angle for each volume. Their significant correlations suggest that the results follow the anatomical knowledge that the fiber orientations are different inside and outside LV.

\subsection{3-D Visualization of Fibers}

We performed 3-D visualization using open-source software ParaView 5.3. $0^{21}$ for each registered volume to qualitatively compare the fiber trajectories from the RCT and $\mu \mathrm{CT}$ volumes in the entire LV. All the points of the trajectories were colored to show the inclination angle. We showed all the tracking results. We trimmed them and showed whether for the sagittal slices, the tracking was done properly in the entire LV. Since ParaView crashed when we directly opened the RCT or $\mu \mathrm{CT}$ volumes, we downsampled these volumes twice by cubic interpolation before opening them.

\section{Results}

\subsection{Fiber Orientation Statistics}

Figure 7 shows the mean and standard deviation of the angle differences of $\mu \mathrm{CT}$ from RCT on axial slices throughout the LV, most of which had mean angle differences of $\mu \mathrm{CT}$ from RCT around $20 \mathrm{deg}$. For instance, their mean and standard deviations were $21.8 \pm 20.5 \mathrm{deg}$ on an axial slice around the central part. Figure 8 shows the angle differences of $\mu \mathrm{CT}$ from RCT on a manually selected slice (depth $=8.85 \mathrm{~mm}$. see Fig. 7). In Fig. 8, fiber orientations at a sample point are represented as two cylinders. A white cylinder shows the fiber orientation estimated from the RCT volume. The colored cylinder shows fiber orientation estimated from the $\mu \mathrm{CT}$ volume, colored based on their angle difference of $\mu \mathrm{CT}$ from RCT.

Figure 9 also shows the estimated fiber orientations. Cylinders show estimated fiber orientation, colored based on their inclination angles.

The relationship of the inclination angles measured in the RCT and $\mu \mathrm{CT}$ volumes is shown in Fig. 10. Each circle in Fig. 10 is gray-scale coded based on the outside ratio. The inclination angles estimated from the RCT and $\mu \mathrm{CT}$ volumes had a correlation coefficient (CC) of 0.63 . No significant difference was observed by Spearman's significant test: $p<2.2 \times 10^{-16}$. This shows $\mu \mathrm{CT}$ produced fiber analysis results that resembled those of RCT. The inclination angles of RCT and the outside ratio also show a significant correlation: $p=2.4 \times 10^{-6}$ with a CC of -0.48 . 


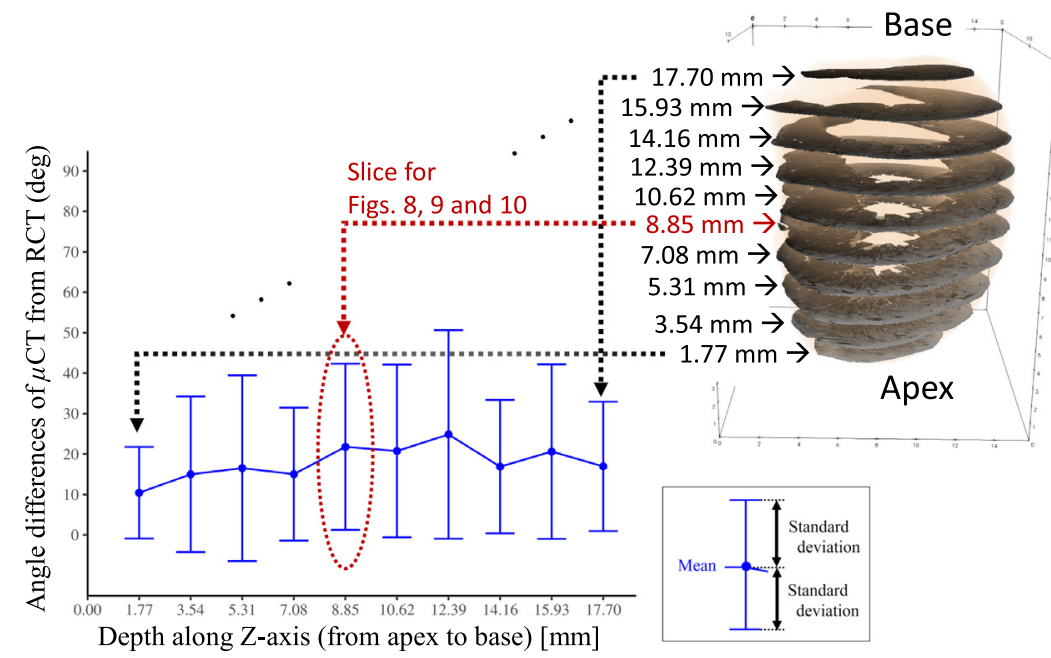

Fig. 7 Mean and standard deviation of angle differences of $\mu \mathrm{CT}$ from RCT at sample points on each axial slice: target axial slices were selected at 100 -slice intervals along $Z$-axis (longest axis from apex to base) of RCT and $\mu \mathrm{CT}$ volumes. Each point on graph shows mean angle differences of $\mu \mathrm{CT}$ from RCT of a sample point on slice, and error bars represent standard deviation. Sample points on each slice were defined by a radial search scheme, explained in Sec. 4.2. Results do not greatly vary throughout the entire LV. Slice located at depth $8.85 \mathrm{~mm}$ is used for further evaluation in Figs. 8-10 and indicated by arrow.
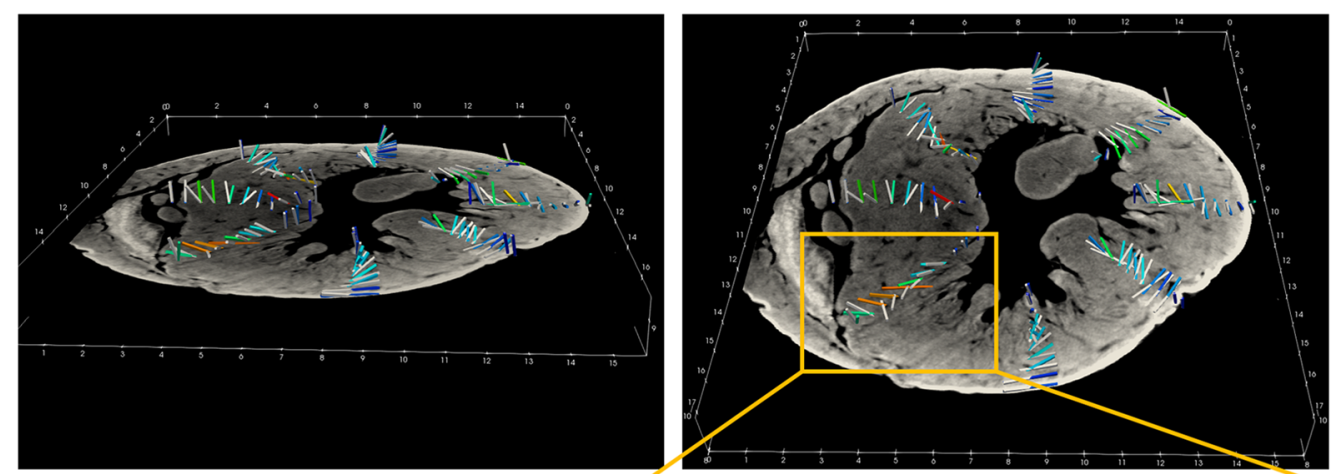

Each colored cylinder is colored based on angle difference of $\mu \mathrm{CT}$ from RCT.
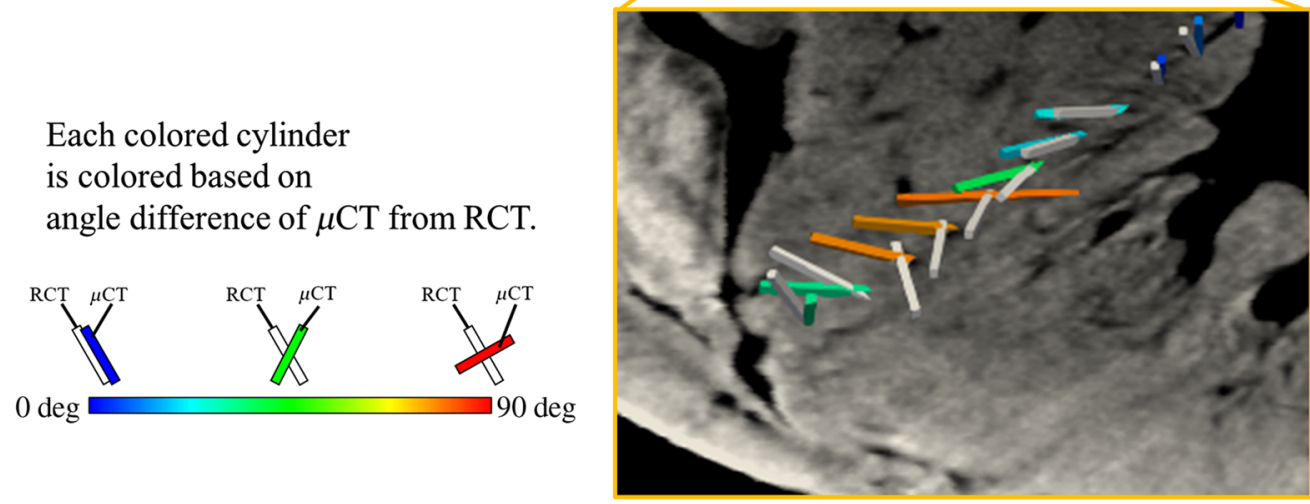

Fig. 8 Angle differences of $\mu \mathrm{CT}$ from RCT on manually selected slice (depth $=8.85 \mathrm{~mm}$, see Fig. 7): colored cylinders show fiber orientations estimated from $\mu \mathrm{CT}$ volume, colored based on angle difference of $\mu \mathrm{CT}$ from RCT. Fiber orientations estimated from RCT volume are also shown as white cylinders. 


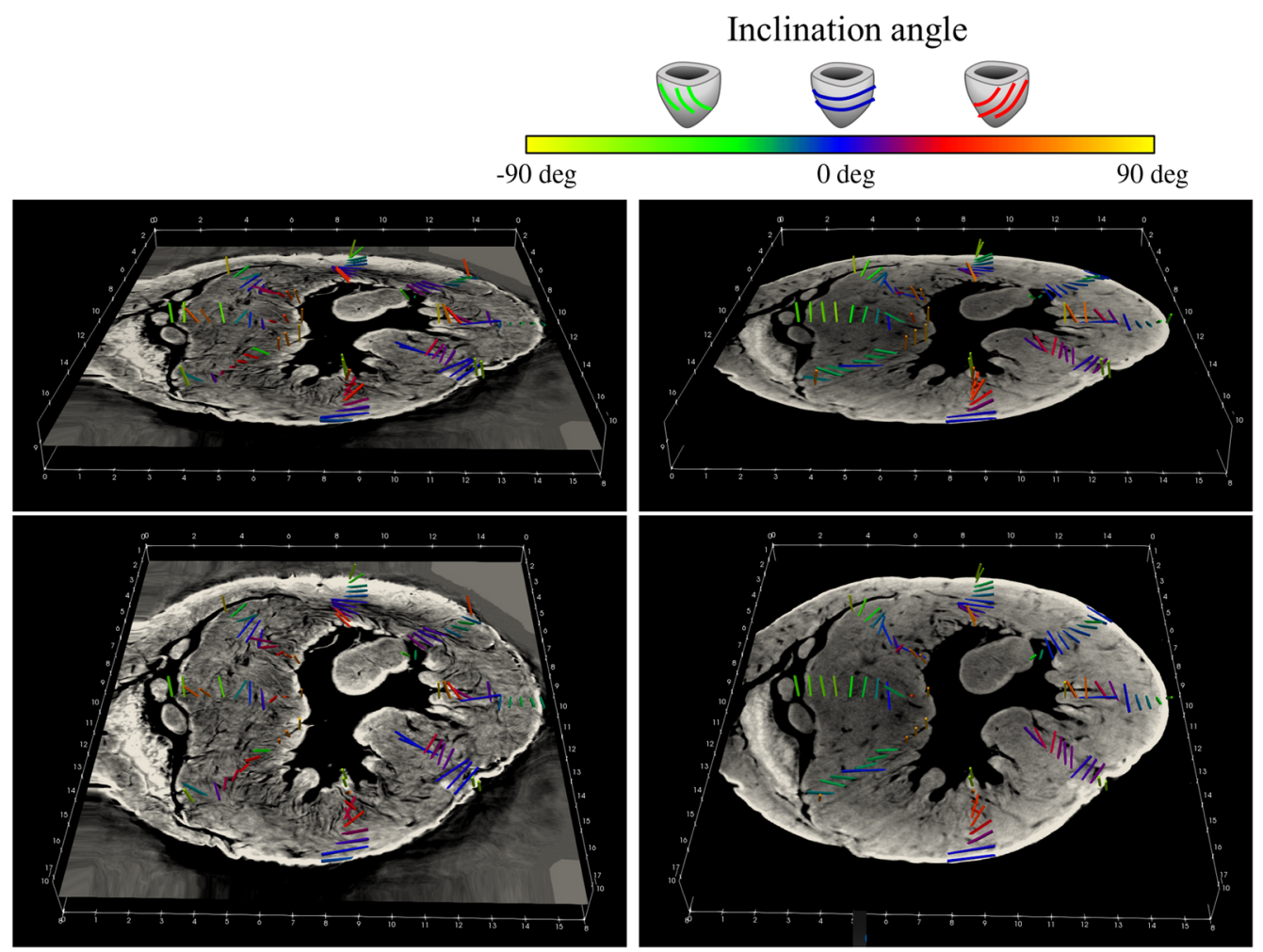

(a) RCT

(b) $\mu \mathrm{CT}$

Fig. 9 Fiber orientations on manually selected slice (depth $=8.85 \mathrm{~mm}$. see Fig. 7) with coloring based on inclination angle: (a) RCT and (b) $\mu \mathrm{CT}$. Cylinders show estimated fiber orientations; colors represent inclination angles.

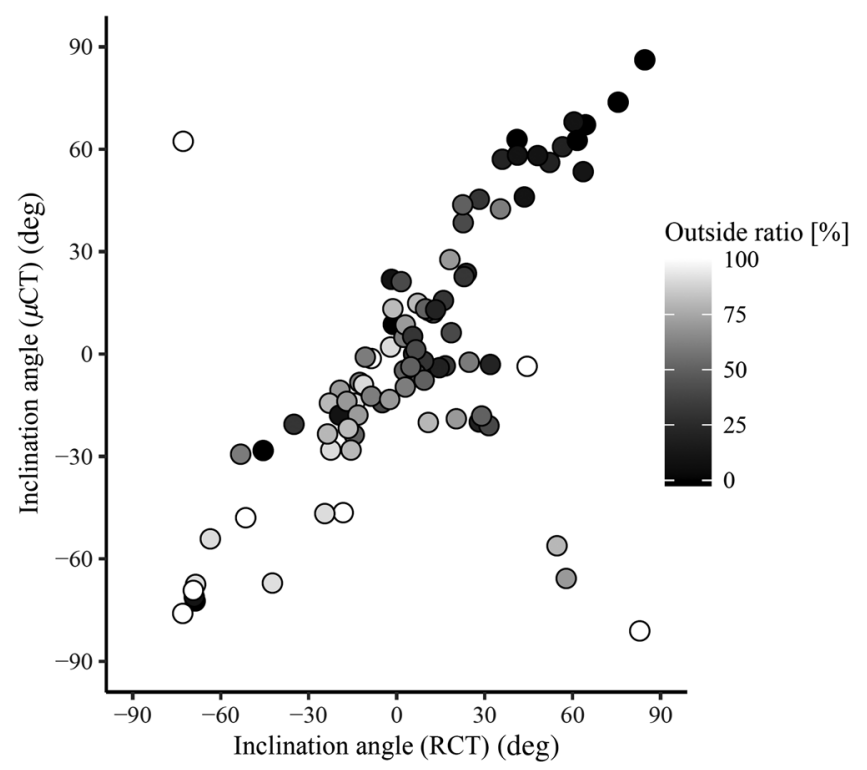

Fig. 10 Relationship of inclination angles measured in RCT and $\mu \mathrm{CT}$ volumes. We manually selected slice (depth $=8.85 \mathrm{~mm}$, see Fig. 7) and plot inclination angles measured on selected slice in this figure. Each circle is gray-scale coded based on outside ratio. Positive correlation is clearly observed between inclination angles estimated from RCT and $\mu \mathrm{CT}$ volumes. We can also observe positive inclination angles in outside area (epicardium area). 
Those of the $\mu \mathrm{CT}$ and the outside ratio are $p=1.2 \times 10^{-7}$ and showed a correlation with a CC of -0.53 .

\subsection{3-D Visualization of Fibers}

Figure 11 shows the fiber trajectories cropped along the coronal plane and a sagittal slice of the $\mu \mathrm{CT}$ or RCT volumes. Colors showing the inclination angles are red inside the LV and green outside it. These color tendencies visually confirm the correspondence of the outside ratio and the inclination angles. However, from the $\mu \mathrm{CT}$ results [Fig. 11(b)], some fiber tracking results were flat and densely gathered. This tendency was not observed in the RCT results [Fig. 11(a)]. These incorrect tracking results from the $\mu \mathrm{CT}$ volume were caused by the joints produced by the scanning procedure, as explained in Sec. 3.1.
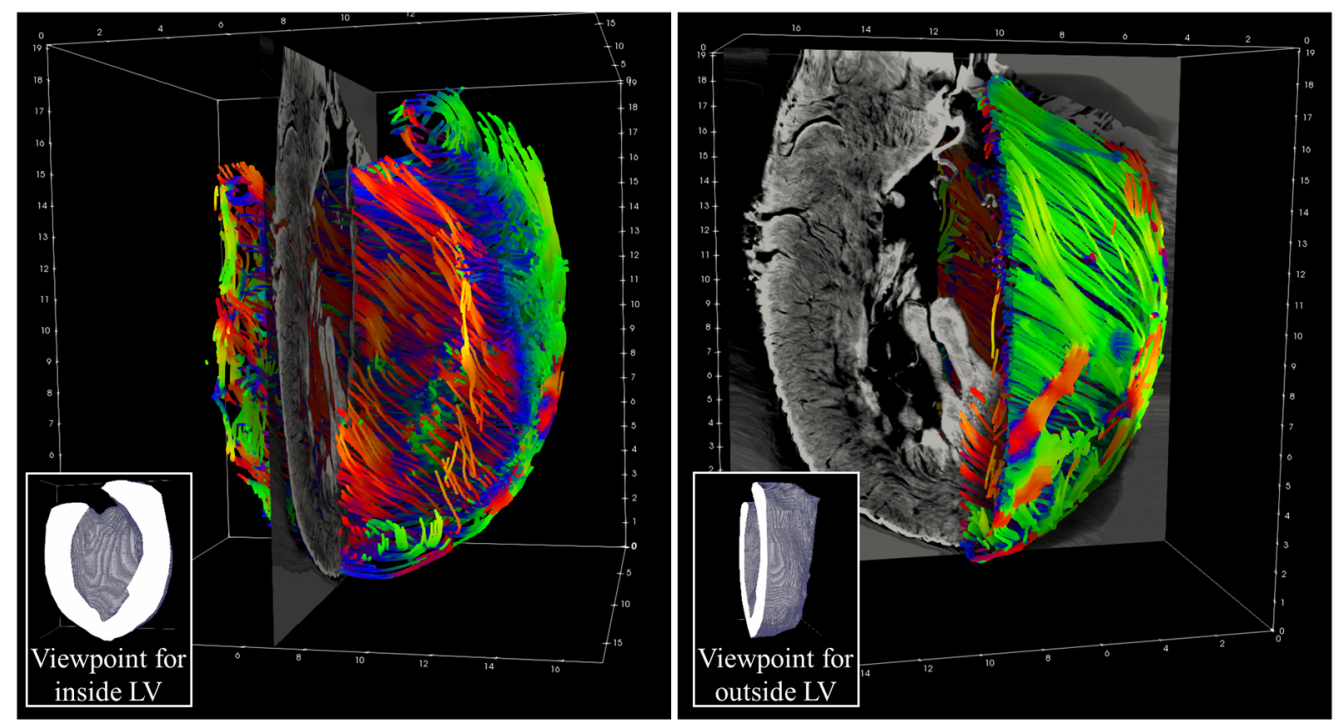

(a) RCT

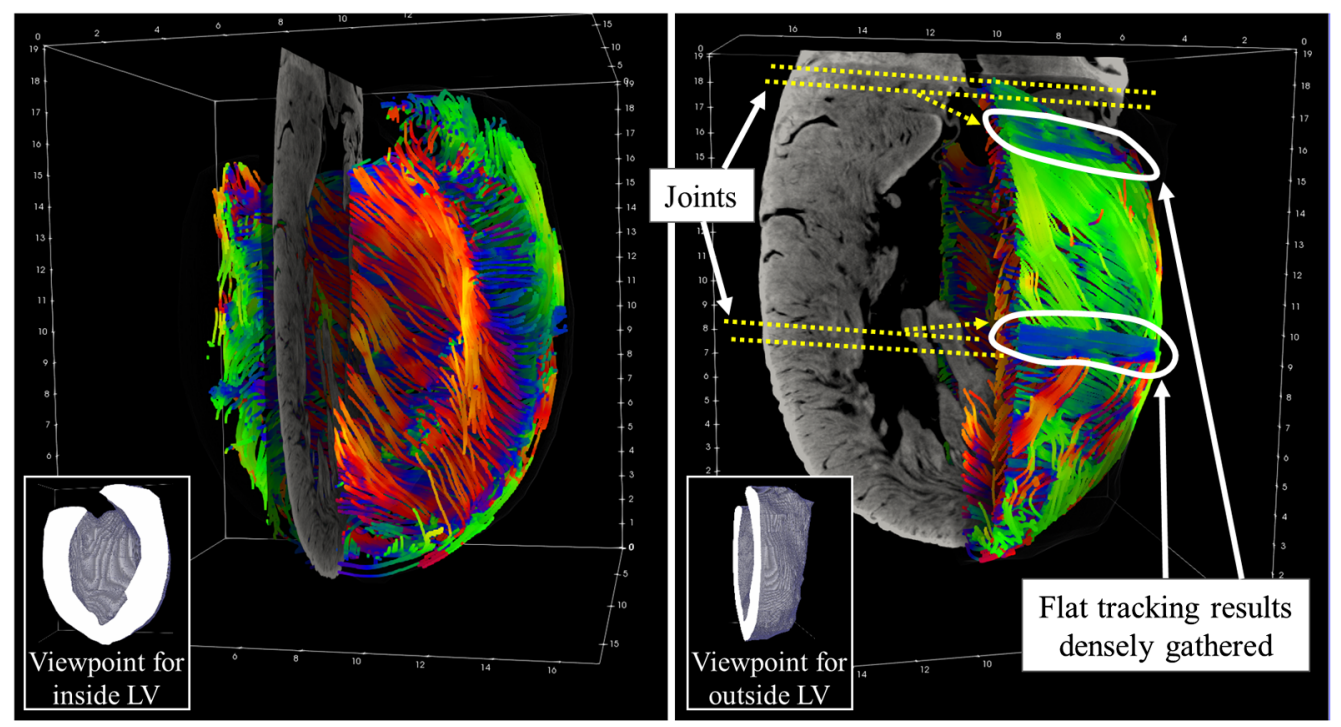

(b) $\mu \mathrm{CT}$

Fig. 11 Fiber tracking results with sagittal slice. Colors represent inclination angles. Two viewpoints were defined: one for observing endocardium and another for epicardium. (a) RCT: tracking was performed properly in entire LV. (b) $\mu \mathrm{CT}$ : although closely resembling RCT results in (a), flat tracking results, densely gathered in joints, were produced due to scanning procedure, explained in Sec. 3.1. 


\section{Discussion}

\subsection{Fiber Orientation Statistics}

The $\mu \mathrm{CT}$ visually had lower contrast for the heart shown in Figs. 3 and 5. The fiber orientation estimations were not very similar to those of the RCT volume, which had an average error around $20 \mathrm{deg}$ (Fig. 7). The average error values were increased by outliers, like the red bars in Fig. 8.

In the part magnified in Fig. 8, many outliers are observed. These errors were caused by an iodine solution artifact (having a higher absorption of $\mathrm{x}$-ray) used for contrast enhancement of $\mu \mathrm{CT}$ imaging (Fig. 8). This iodine solution created a strong artifact in a slice plane. A tracking algorithm traced it and produced in-plane (flat) tracking.

The colors of the points in Fig. 9 suggest that the inclination angles computed from both the $\mathrm{RCT}$ and $\mu \mathrm{CT}$ volumes were positive inside and negative outside the LV. This tendency was already proved through anatomical studies, ${ }^{5}$ and the results of both the $\mu \mathrm{CT}$ and RCT volumes followed it.

We used nonrigid registration to compensate for the deformation of the specimen at the RCT and $\mu \mathrm{CT}$ scanning times. Our scanning procedures were performed in the following order: RCT scanning, iodine staining, and $\mu \mathrm{CT}$ scanning, as explained in Sec. 3.1. Iodine staining caused a slight contraction of the heart. There were some changes in the specimen sizes and small structures between the two CT volumes.

\subsection{3-D Visualization of Fibers}

Fiber tracking allows intuitive understanding of fiber running orientations in 3-D space. The tendency of inclination angles, correlated to the outside ratio, was also visually observed in the fiber tracking results from both the RCT and $\mu \mathrm{CT}$ volumes (Fig. 11). Most of the fiber tracking results inside the LV were red, and most of those outside were green. The trajectories were visually smooth from both volumes.

Figure 11 shows the fiber tracking results from the base to the apex. One large difference between the RCT and $\mu \mathrm{CT}$ volumes is apparent. On the results from the $\mu \mathrm{CT}$ volume [Fig. 11(b)], flat tracking results are densely gathered. Since our $\mu$ CT scanner had a limited FOV, the rabbit heart was scanned by dividing it into three parts (Sec. 3.1). Since the images of the three scanning results were not precisely aligned, their joints were followed by tracking. Correction processes for such mistracking are required in the future.

We found that it is possible to estimate fiber orientation well on $\mu \mathrm{CT}$ volumes. Our fiber orientation estimation procedures were useful for fiber tracking in the entire LV, although the results must be scrutinized for errors between two scans. $\mu \mathrm{CT}$, which is a promising modality for cardiac imaging and useful for observing cardiac fibers, is commonly used by many companies and institutes for industrial purposes. Our work shows an application for cardiac imaging, which presents imaging protocols and their usefulness for observing cardiac fibers.

\section{Conclusions and Limitations}

We described our fiber analysis methods from the RCT or $\mu \mathrm{CT}$ volumes of the heart and analyzed how $\mu \mathrm{CT}$ produces proper results using our methods by comparing them with RCT. A rabbit heart was fixated by ethanol, scanned by RCT, stained in an iodine solution, and scanned by $\mu \mathrm{CT}$. The RCT and $\mu \mathrm{CT}$ volumes were nonlinearly registered. The fiber orientation of each point was estimated using the ST analysis of each volume. We defined two measures, the angle difference of $\mu \mathrm{CT}$ from RCT and inclination angles, to compare the fiber orientation estimation results at the sample points of these volumes. Although promising results were obtained in the cardiac fiber analysis using $\mu \mathrm{CT}$, we need to perform further investigation on the differences of the results obtained from the $\mu \mathrm{CT}$ and RCT volumes. Analysis results from both modalities match well the anatomical knowledge that fiber orientations are different inside and outside of the LV. Unfortunately, the $\mu \mathrm{CT}$ volume caused incorrect tracking around the boundaries of the scanning of the stitching. Smoothing around the boundaries is our future work. 
Our work suffers from the following limitations. First, just one specimen is inadequate for comparison experiments of two scans and their analysis. However, this problem is caused by restricted usage of RCT scanning that needs to use the synchrotron facility (circumference: $187 \mathrm{~m}$ ) shared by worldwide high-energy physics researchers. Obtaining beam time is difficult and expensive. This is why we have only one sample. Increasing the number of samples is also future work. Second, the quantitative validation of fiber orientation results is required from a RCT volume that has been used as ground-truth. Several manually set parameters and the evaluation of different sets of parameters are also needed. One idea is a comparison with histopathological sections, but such a project is very complicated, as explained in Sec. 1. Therefore, future work will include deeper validation using many more hearts to quantitatively validate the fiber estimation of SRs for RCT volumes. We would also like to find ways to observe not only fibers in the LV but also other parts and tissues in the heart.

\section{Disclosures}

No author involved with this paper has any conflict of interest.

\section{Acknowledgments}

Parts of this work were supported by MEXT/JSPS KAKENHI (Grant Nos. 26108006, 17H00867, 17K20099, 16H05427, 17H05288), the JSPS Bilateral Joint Research Project, AMED (19lk1010036h0001), and the Hori Sciences and Arts Foundation.

\section{References}

1. E. J. Benjamin et al., "Heart disease and stroke statistics-2018 update: a report from the American Heart Association," Circulation 137, e67-e492 (2018).

2. P. J. Basser et al., "In vivo fiber tractography using DT-MRI data," Magn. Reson. Med. 44(4), 625-632 (2000).

3. P. Helm et al., "Measuring and mapping cardiac fiber and laminar architecture using diffusion tensor MR imaging," Ann. N.Y. Acad. Sci. 1047(1), 296-307 (2005).

4. D. Rohmer, A. Sitek, and G. T. Gullberg, "Reconstruction and visualization of fiber and laminar structure in the normal human heart from ex vivo diffusion tensor magnetic resonance imaging (DTMRI) data," Invest. Radiol. 42(11), 777-789 (2007).

5. D. D. Streeter, Jr. et al., "Fiber orientation in the canine left ventricle during diastole and systole," Circ. Res. 24(3), 339-347 (1969).

6. D. F. Scollan et al., "Histological validation of myocardial microstructure obtained from diffusion tensor magnetic resonance imaging," Am. J. Physiol. 275(6), H2308-H2318 (1998).

7. R. Casero et al., "Transformation diffusion reconstruction of three-dimensional histology volumes from two-dimensional image stacks," Med. Image Anal. 38, 184-204 (2017).

8. N. Sunaguchi et al., "X-ray refraction-contrast computed tomography images using darkfield imaging optics," Appl. Phys. Lett. 97(15), 153701 (2010).

9. N. Sunaguchi et al., "In vitro validation of an artefact suppression algorithm in x-ray phasecontrast computed tomography," PLoS One 10(8), e0135654 (2015).

10. H. Oda et al., "Scanning, registration, and fiber estimation of rabbit hearts using micro-focus and refraction-contrast x-ray CT," Proc. SPIE 10953, 109531I (2019).

11. M. Varela, J. Zhao, and O. V. Aslanidi, "Determination of atrial myofibre orientation using structure tensor analysis for biophysical modelling," Lect. Notes Comput. Sci. 7945, 425-432 (2013).

12. O. V. Aslanidi et al., "Application of micro-computed tomography with iodine staining to cardiac imaging, segmentation, and computational model development," IEEE Trans. Med. Imaging 32(1), 8-17 (2013).

13. T. Kunii et al., "Visualization of rat myocardial layers in phase-contrast $x$-ray imaging with ethanol fixation," Med. Imaging Technol. 31(2), 132-135 (2013). 
14. High Energy Accelerator Research Organization, https://www.kek.jp/en/Facility/IMSS/PF/ PFRing/Imaging/.

15. Ministry of Education, Culture, Sports, Science and Technology-JAPAN, http://www.mext .go.jp/b_menu/shingi/chousa/shinkou/010/houkoku/05081201/s001.htm.

16. D. Gürsoy et al., "TomoPy: a framework for the analysis of synchrotron tomographic data," J. Synchrotron Radiat. 21(5), 1188-1193 (2014).

17. R. S. Stephenson et al., "Contrast enhanced micro-computed tomography resolves the 3-dimensional morphology of the cardiac conduction system in mammalian hearts," PLoS One 7(4), e35299 (2012).

18. M. Nolden et al., "The medical imaging interaction toolkit: challenges and advances," Int. J. Comput. Assisted Radiol. Surg. 8(4), 607-620 (2013).

19. M. P. Heinrich et al., "MRF-based deformable registration and ventilation estimation of lung CT," IEEE Trans. Med. Imaging 32(7), 1239-1248 (2013).

20. H. Lombaert et al., "Statistical analysis of the human cardiac fiber architecture from DT-MRI," Lect. Notes Comput. Sci. 6666, 171-179 (2011).

21. U. Ayachit, The ParaView Guide: A Parallel Visualization Application, Kitware, Inc., New York (2015).

Hirohisa Oda received his MEng degree from Nagoya University in 2014. After working in industry, he started a PhD program of Nagoya University from 2015. His specializations are image processing and machine learning. His research interests are computer-aided diagnosis and microfocus $\mathrm{x}$-ray CT for cardiac image processing.

Holger R. Roth has been a senior applied research scientist at NVIDIA's deep learning for medical imaging research group based in Bethesda, Maryland, USA, since 2018. Previously, he was an assistant professor (research) at Mori Laboratory, Nagoya University, Japan, and visiting research fellow at the National Institutes of Health Clinical Center, USA, after graduating with a $\mathrm{PhD}$ from the University College London, United Kingdom. His current research focuses on the application of deep learning methods for large-scale medical image analysis, multiscale computational anatomy, and computer-aided detection and diagnosis. He is also an associate editor for IEEE Transactions on Medical Imaging.

Takaaki Sugino received his $\mathrm{PhD}$ from the Graduate School of Engineering, Chiba University in 2017. From 2017 to 2019, he was a research associate in the Graduate School of Informatics, Nagoya University. He is currently an assistant professor in the Department of Biomedical Information, Tokyo Medical and Dental University. His research interests are computer-assisted interventions and medical image processing.

Naoki Sunaguchi graduated from the Faculty of Engineering, Yamagata University, Japan, in 2004. He received his $\mathrm{PhD}$ in engineering from Yamagata University, Japan, in 2010. He is currently an associate professor at the Graduate School of Medicine, Nagoya University, Japan. His research interests include x-ray imaging techniques and reconstruction algorithms based on the phase contrast $\mathrm{x}$-ray computed tomography. He has developed $\mathrm{x}$-ray dark-field imaging system to observe biomedical soft tissue in high image quality.

Noriko Usami is a technician at the Graduate School of Informatics, Nagoya University. She graduated from the Faculty of Medical Engineering, Suzuka Medical University, in 2001.

Masahiro Oda is an assistant professor at the Graduate School of Informatics, Nagoya University. In 2004, he enrolled at the Department of Media Science, Graduate School of Information Science, Nagoya University. In 2009, he received his PhD from the Department of Media Science, Graduate School of Information Science, Nagoya University. His research interests are medical image processing, computer-aided diagnosis, and computer-assisted interventions.

Daisuke Shimao is a professor at the Department of Radiological Technology, Faculty of Health Sciences, Hokkaido University of Science. He received his PhD from the Graduate University 
for Advanced Studies in 2006. His research interests are clinical imaging technology and image anatomy.

Tetsuya Yuasa has been with the Yamagata University, Yonezawa, Japan, from 1991, where he is now a professor at the Department of Bio-Systems Engineering. He received his PhD in mathematical engineering and information physics from the University of Tokyo, Japan, in 1997. His research interests are CT imaging methods based on various kinds of physical probes such as near-infrared laser light, electron spin resonance, $\mathrm{x}$-ray, and synchrotron X-ray, as well as their data/image processing.

Toshiaki Akita is a designated professor at the Department of Cardiac Surgery, Nagoya University Hospital. He received his $\mathrm{MD}$ and $\mathrm{PhD}$ degrees in medicine from the Graduate School of Medicine, Nagoya University in 1982 and 1994, respectively. His research interest is the development of the device treatment of congestive heart failure.

Kensaku Mori is a professor of Information and Communications and Graduate School of Information Science, Nagoya University, and the director of Information Technology Center of Nagoya University. He is also MICCAI fellow. He is the president of the International Society for Computer-aided Surgery. He received his MEng (information engineering) and $\mathrm{PhD}$ (information engineering) from Nagoya University in 1994 and 1996. He started his academic carrier in 1997 as an assistant professor at Nagoya University. Then he was promoted as associate professor in 2001 at Nagoya University. In 2009, he obtained full professorship at Nagoya University. In 2016, he was appointed as the director of the Information Technology Center of Nagoya University. In 2017, he was appointed as a professor of Department of Intelligent Systems, Graduate School of Informatics. He is involved in many international conference organizations, including SPIE Medical Imaging, CARS, and MICCAI, as a general chair or program committee member. He was a conference co-chair of SPIE Medical Imaging Computer Aided Diagnosis conference in 2018 and 2019. He is also an editorial board member of SPIE's Journal of Medical Imaging.

Biographies of the other authors are not available. 